\title{
Emifiermiernta
}

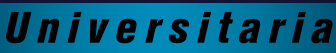

\section{Biblioteca Virtual en Salud (BVS) México Enfermería. Un espacio construido por y para la enfermería mexicana}

\section{Virtual Health Library (VHL) Mexico Nursing. A space built by and for the Mexican nursing}

\section{Biblioteca Virtual em Saúde Enfermagem (BVS) do México. Um espaço construído por e para a enfermagem mexicana}

A 20 años del establecimiento de la Biblioteca Virtual de Salud (BVS), como modelo, estrategia y plataforma operacional de cooperación técnica de la Organización Panamericana de la Salud (OPS), para la gestión de la información y del conocimiento en salud en la Región de América Latina y el Caribe (AL\&C), hoy día, este espacio es una Red de Redes construida colectivamente coordinada por el Centro Latinoamericano y del Caribe de Información en Ciencias de la Salud, el cual es un organismo especializado de la OPS (BIREME/OPS/OMS), con la misión de contribuir en el desarrollo de la salud en los países de AL\&C, por medio de la democratización del acceso, publicación y uso de información, conocimiento y evidencia científica ${ }^{1}$.

Esta iniciativa se desarrolla, en principio, de modo descentralizado por medio de instancias nacionales (BVS Argentina, BVS Brasil, BVS México etc.); posteriormente, a través de redes temáticas de instituciones relacionadas con la investigación, con la enseñanza, con los servicios o bien con aspectos de particular interés para los países o los profesionales de la salud (BVS Enfermería, BVS Ministerios de Salud, etc.).

Es importante señalar, que la base para concretar los diferentes esfuerzos es a través del trabajo en Red, como atinadamente fue señalado por el Dr. Diego González, actual Director de BIREME, durante la $7^{a}$ Reunión de Coordinadores de Centros Cooperantes para la Red BVS, que se llevó a cabo en diciembre 2018, en Sao Paulo, Brasil (BVS7), donde, quienes participamos, tuvimos la oportunidad de reflexionar respecto a la contribución de la información y la evidencia en la agenda de Salud Sostenible 2018-2030, para la Américas, así como la importancia de contar con espacios donde prevalezca el acceso abierto que posibiliten el uso y aplicación de manera consciente y responsable de la evidencia científica. Esto sin perder de vista, que si bien la evidencia es global, su aplicación debe ser siempre local, así como considerar el contexto que prevalece en cada país, Institución o situación particular.

Para el caso de la Enfermería Latinoamericana es a partir del año 2009, que se dan los primeros trabajos hacia la construcción de la Red Temática BVS Enfermería, con la misión de la construcción del patrimonio informacional, con fácil acceso, sin limitaciones por el tiempo, espacio ni fronteras, además buscar e impulsar los procesos de generación de conocimiento y la contribución para la formación y la 
práctica de la enfermería, para actuar con el compromiso ético-social en el área de la educación, la investigación y la atención a la salud, así como para elevar la calidad de vida de la población en Brasil y en la Región ${ }^{2}$.Estos esfuerzos se concretaron en el año 2012, a partir de la creación del espacio virtual BVS Enfermería Regional (http://bvsenfermeria.bvsalud.org/), al cual de manera gradual grupos de trabajo de enfermería de diversos países se han ido incorporando con su propia producción de conocimientos, en espacios creados ex profeso, a través de diversos mecanismos de colaboración al interior de los mismos países, con el apoyo invaluable de la OPS y de la BIREME.

Respecto a nuestro país, es a mediados del año 2014, que formalmente tuvimos los primeros acercamientos para sumarnos a esta iniciativa, a través de la Red Mexicana de Editores de Revistas Científicas de nuestro país (Red MERCE), un grupo que asumió (y lo sigue haciendo) entre sus prioridades ${ }^{3}$ dar los primeros pasos para la construcción de la BVS-Enfermería México a través de la indización del acervo, por lo menos de los últimos cinco años, con el que cada Revista integrante de la Red contaba en ese momento (esto es un compromiso permanente pues cada número que aparece debe ser incluido en esta base de datos). Lo anterior hizo necesarias una serie de tareas, algunas de manera paralela conforme el proyecto avanzaba, que implicaron, entre otras cosas, la vinculación y comunicación con el Ing. Dante Salazar responsable de éste y otros proyectos dentro de la Representación de la OPS México, con la BIREME en Brasil, para la aprobación del proyecto y la asignación de un espacio dentro de la BVS Regional, la capacitación de manera virtual a las integrantes de la Red MERCE y los grupos de trabajo en el sistema que en ese momento se utilizaba para la indización de documentos (en este caso de artículos de investigación) y, por supuesto, una amplia gestión al interior de las Instituciones de cada una de las Revistas, para dar a conocer el proyecto y éste pudiese echarse a andar.

Una vez que se avanzó en esta primera etapa, se tomó la decisión, que era necesario, en segundo lugar, dar a conocer (por su potencial contribución al cuidado en la práctica): las Guías de Práctica Clínica en Enfermería (GPCE) elaboradas por estos profesionales de nuestro país. Para ello, nos vinculamos con la Titular de la Dirección de Enfermería y Coordinadora de la Comisión Permanente de Enfermería (CPE) quien, a través de la Mtra. María de Jesús Posos González, responsable operativa del proyecto dentro de la propia CPE, al conocer el proyecto lo adoptó de manera entusiasta junto con el Lic. José Luis Galindo Ortega (Jefe del Centro de Información y Documentación Científica en el Instituto Nacional de Pediatría), para la indización de las GPCE existentes en ese momento, de igual manera con el compromiso de incluir dentro de la base aquellas que se vayan elaborando.

Actualmente, el proyecto inicia su tercera etapa con la inclusión de Tesis de los distintos Programas de Maestría en Enfermería que se llevan a cabo en nuestro país, particularmente los que están incluidos dentro del Padrón de Programas de Calidad del CONACyT. Las primeras son aquellas del Programa de Maestría de la UNAM, pero seguramente, de forma gradual se irán sumando muchos otros Programas, que permitan el acercamiento al conocimiento vasto que se produce en estos ámbitos, pero que, desafortunadamente, poco se conoce.

Dentro de esta espiral ascendente, también ha sido necesaria la capacitación en el nuevo sistema de indización creado por BIREME, para la inclusión de nuevos documentos y un hecho muy particular es la renovación del Portal Regional de la BVS, el cual se dio a conocer el pasado mes de diciembre, nos enorgullecemos de contar ya, en este sitio dentro de la Red Temática de esta disciplina, con la propia página de la BVS Enfermería de México (https://bit.ly/2Myj6LA). Este hecho trascendente, que nos congratulamos presentar, señala el siguiente paso a dar, que no es el último, difundir este sitio en diferentes medios y espacios a fin de alcanzar uno de los objetivos más importantes de todo este trabajo, el uso y aplicación de esta información producida por y para los profesionales de la enfermería en México, es entonces cuando estaremos seguros de lo que esta contribución representa para nuestro país. 


\section{Referencias}

1. BIREME/OPS/OMS. Portal Regional de la BVS: Acerca de la BVS. São Paulo - SP: Centro Latinoamericano y del Caribe de Información en Ciencias de la Salud. [Consultado diciembre 14 2018]. Disponible en: https://bit.ly/2Hy1d0C

2. Felix-Lana FC. BVS enfermagem e as novas perspectivas [Editorial]. REME 2009; 13(1); 11. [Consultado diciembre 8 2019]. Disponible en https://bit.ly/2WgxAUO

3. Matus-Miranda R. La Red Mexicana de Editores de Revistas Científicas de Enfermería y su responsabilidad en la difusión del conocimiento. Rev Enferm Inst Mex Seguro Soc. 2017; 25 (4):2413. [Consultado diciembre 14 2018]. Disponible en: https://bit.ly/2Topcka

R. Matus Miranda

ORCID: 0000-0001-6581-7263

Escuela Nacional de Enfermería y Obstetricia, Ciudad de México, México Líder del Proyecto BVS Enfermería México Correo electrónico: reyna.matus@gmail.com

3

$\stackrel{\infty}{\infty}$ 